\section{Stem cell transplantation for congenital dyserythropoietic anemia: an analysis from the European Society for Blood and Marrow Transplantation}

Congenital dyserythropoietic anemias (CDA) are a group of heterogeneous disorders characterized by hyporegenerative anemia and ineffective erythropoiesis, with related reticulocytopenia, and iron overload. Specific morphological aspects of late erythroblasts in the bone marrow form the most important, albeit non-specific, feature of the disease. Morphology has always been the most important tool for diagnosis and is still used to classify the disease into three classical forms and variants. ${ }^{2}$ Nonetheless, in the last few years, mutations of six specific genes related to the regulation of DNA and cell division have been identified as being causative..$^{3-5}$

Patients with CDA usually show anemia, jaundice, splenomegaly, ineffective erythropoiesis and the typical marrow features. Somatic abnormalities involving fingers and nails can be part of the clinical features.

The management of the disease is generally limited to blood transfusion and iron chelation. Interferon- $\alpha$ treat- ment has also been successfully used in patients with CDA type $I^{6,7}$ whereas splenectomy has been proved to reduce the number of transfusions in CDA II. ${ }^{8}$

Overall, the prognosis of CDA patients is good; ${ }^{2}$ however, stem cell transplantation (SCT) represents the only curative option for this disease. Some reports have shown its efficacy, but data from the literature are scarce and limited to a very small number of patients, mostly transplanted from sibling donors. ${ }^{9-16}$

In this retrospective study, we describe the outcome of SCT in a large cohort of patients with CDA. The study was conducted on behalf of the Severe Aplastic Anemia Working Party (SAAWP) of the European Society for Bone and Marrow Transplantation (EBMT) and relied on data from patients affected with CDA who underwent SCT and were registered in the EBMT Database. Clinical information on the disease was collected by a questionnaire distributed to participating centers and the details on transplant procedures were obtained by analyzing the database.

Engraftment was defined as neutrophil count $>0.5 \mathrm{x}$ $10^{9} / \mathrm{L}$ for at least three consecutive days. Primary graft failure was defined as neutrophil count never reaching $>0.5 \times 10^{9} / \mathrm{L}$ and secondary graft failure was defined as a
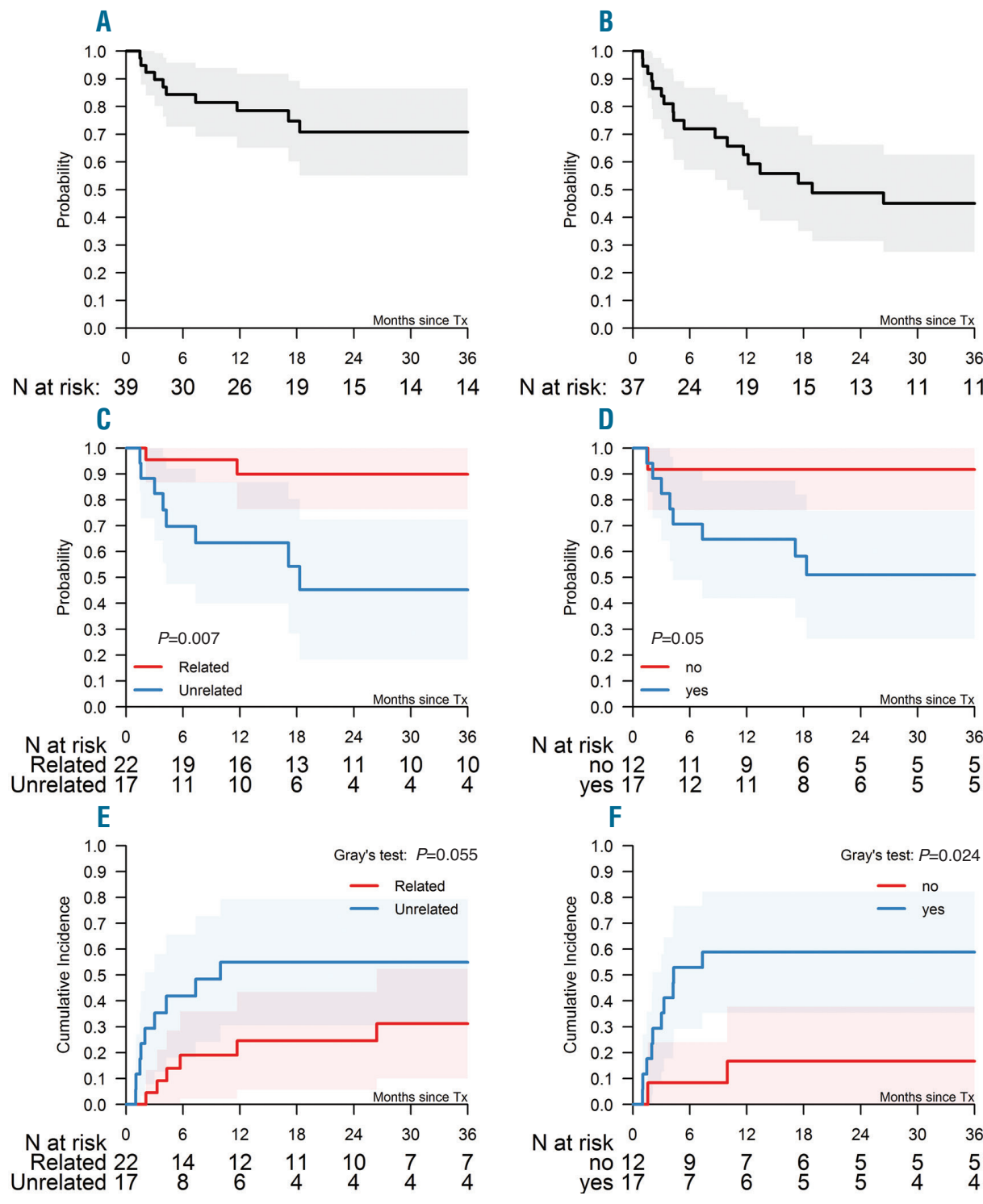

Figure 1. Outcome of patients with congenital dyserythropoietic anemias (CDA) undergoins stem cell transplantation (SCT). (A) Overall survival (OS). (B) Event-free survival. (C) OS according to donor type. (D) OS according to iron overload. (E) Graft failure incidence according to donor type. (F) Graft failure incidence according to iron overload. 


\section{LETTERS TO THE EDITOR}

Table 1. Patients' characteristics and transplant features.

\begin{tabular}{|c|c|c|c|c|}
\hline Patients'characteristics & $\mathrm{N}=39$ & SCT from sibling & SCT from MUD & 36 months OS \\
\hline \multicolumn{5}{|l|}{ CDA sub-types } \\
\hline type I & $10(26 \%)$ & 8 & 2 & $80 \%$ \\
\hline type II & $13(33 \%)$ & 5 & 8 & $61 \%$ \\
\hline type IV & $1(3 \%)$ & 0 & 1 & \\
\hline xltda (gata-1) & $1(3 \%)$ & 0 & 1 & \\
\hline Missing & $14(35 \%)$ & & & \\
\hline \multicolumn{5}{|l|}{ Molecular mutation } \\
\hline SEC23B & $6(15 \%)$ & 2 & 4 & \\
\hline GATA-1 & $2(5 \%)$ & 1 & 1 & \\
\hline CDAN1 & $1(3 \%)$ & 1 & 0 & \\
\hline No mutation & $24(62 \%)$ & 14 & 10 & \\
\hline Missing & $6(15 \%)$ & 4 & 2 & \\
\hline Somatic abnormalities & $8 / 39(20 \%)$ & & & \\
\hline Skeletal & 4 & 2 & 2 & \\
\hline Skin & 1 & 0 & 1 & \\
\hline Neurological & 3 & 1 & 2 & \\
\hline Retina & 0 & 0 & 0 & \\
\hline Dysmorphic face & 1 & 1 & 0 & \\
\hline Macrocephaly & 1 & 1 & 0 & \\
\hline Pre-transplant treatment & $20(51 \%)$ & & & \\
\hline Splenectomy & $2(5 \%)$ & 0 & 2 & \\
\hline Chelation treatment & $13(33 \%)$ & 7 & 6 & \\
\hline IFN & $5(13 \%)$ & 1 & 4 & \\
\hline \multicolumn{5}{|l|}{ Clinical manifestation at SCT } \\
\hline Hepatomegaly & $15(38 \%)$ & 9 & 6 & $77 \%$ \\
\hline Jaundice & $9(23 \%)$ & 6 & 3 & $41 \%$ \\
\hline Splenomegaly & $18(46 \%)$ & 12 & 6 & $68 \%$ \\
\hline Iron overload & $17(43 \%)$ & 8 & 9 & $51 \%$ \\
\hline Age at HSCT, years (median, range) & $5.1(0.9-38)$ & $4(1-38)$ & $7(0.9-31)$ & \\
\hline $\begin{array}{l}\text { Time from diagnosis to SCT, } \\
\text { months (median, range) }\end{array}$ & $34.8(2.4-377)$ & $23(2-177)$ & $55(2-377)$ & \\
\hline \multicolumn{5}{|l|}{$\begin{array}{l}\text { Performance status at HSCT } \\
\text { (Lansky or Karnofsky) }\end{array}$} \\
\hline$<90$ & $5(13 \%)$ & 3 & 2 & \\
\hline $90-100$ & $28(71 \%)$ & 16 & 12 & \\
\hline Missing & $6(15 \%)$ & & & \\
\hline \multicolumn{5}{|l|}{ Year of SCT } \\
\hline Before 2010 & $18(46 \%)$ & 11 & 7 & $65 \%$ \\
\hline After 2010 & $21(54 \%)$ & 11 & 10 & $79 \%$ \\
\hline \multicolumn{5}{|l|}{ Donor type } \\
\hline Identical sibling & $20(51 \%)$ & & & $90 \%$ \\
\hline Unrelated donor & $17(44 \%)$ & & & $45 \%$ \\
\hline Other relative & $2 \quad(5 \%)$ & & & \\
\hline \multicolumn{5}{|l|}{ HLA match } \\
\hline $10 / 10$ & 12 & $4(29 \%)$ & $7(47 \%)$ & $66 \%$ \\
\hline$<10 / 10$ & 19 & $10(71 \%)$ & 8 & $58 \%$ \\
\hline Missing & 8 & & & \\
\hline \multicolumn{5}{|l|}{ Stem cell source } \\
\hline Bone marrow & $30(76 \%)$ & 18 & 12 & \\
\hline Peripheral blood & 7 (18\%) & 3 & 4 & \\
\hline Cord blood & $2(5 \%)$ & 1 & 1 & Continu \\
\hline
\end{tabular}




\begin{tabular}{|c|c|c|c|}
\hline Busulfan-based conditioning & $28(72 \%)$ & 18 & 10 \\
\hline Bu-Cy & 10 & 10 & 0 \\
\hline Bu-Cy-ATG & 9 & 6 & 3 \\
\hline Bu-Flu-ATG & 3 & 2 & 1 \\
\hline Bu-Flu-TT & 1 & 0 & 1 \\
\hline Bu-Flu-TT & 1 & 0 & 1 \\
\hline Bu-Flu-Cy-ATG & 2 & 0 & 2 \\
\hline Bu-Flu-LPAM -ATG & 1 & 0 & 1 \\
\hline Bu-Cy-LPAM-ATG & 1 & 0 & 1 \\
\hline Treosulfan-based conditioning & $8(20 \%)$ & 1 & 7 \\
\hline Treo-Flu-TT & 2 & 0 & 2 \\
\hline Treo-Flu-TT-ATG & 4 & 0 & 4 \\
\hline Treo-Flu-LPAM-ATG & 1 & 1 & 0 \\
\hline Treo-Cy-ATG & 1 & 0 & 1 \\
\hline Other conditioning & $3(8 \%)$ & & \\
\hline $\mathrm{Cy}$ & 1 & 2 & 0 \\
\hline Cy-ATG & 1 & 1 & 0 \\
\hline Missing & 1 & & \\
\hline Secondary graft failure & 8 & 4 & 4 \\
\hline Acute GvHD, grade III-IV (100 days) & 11 & 2 & 9 \\
\hline Chronic GvHD (36 months) & & 5 & 2 \\
\hline Limited/Extensive & 7 & & \\
\hline Death & 10 & 2 & 8 \\
\hline Follow up, median months (range) & $44(13-71)$ & & \\
\hline
\end{tabular}

decrease in neutrophil count to a lower level after initial engraftment. Overall survival (OS) and event-free survival (EFS), defined as survival without rejection, graft loss or a second transplant, were calculated using the Kaplan-Meier product limit estimation method; differences in subgroups were assessed by the Log rank test. Median follow up was estimated using the reverse Kaplan-Meier method. Cumulative incidences of grade IIIV acute graft-versus-host disease (aGvHD), of limited/extensive chronic GvHD (cGvHD), and of graft failure were analyzed separately in a competing risks framework, and subgroup differences were assessed by Gray test. Competing events for graft failure, acute/chronic GvHD, include second transplant, relapse and death. All estimates were reported with a corresponding $95 \%$ confidence interval; $P<0.05$ was considered significant. Iron overload was defined as ferritin serum level $>1000 \mathrm{mg} / \mathrm{dL}$ or as the presence of pathological liver iron concentration by magnetic resonance imaging (MRI).

Between 1996 and 2016, 39 patients (22 males), with a median age of 5.1 years (range, 0.9-38.2) underwent SCT following a conditioning regimen that was myeloablative in all but one case. SCT was performed because of transfusion dependency alone $(n=34)$ or associated with iron overload ( $n=5)$. Patients' clinical characteristics are shown in Table 1. Only one patient in this cohort has been previously reported. ${ }^{10}$

All patients were engrafted. The 3-year incidence of secondary graft failure was $12 \%(1-23 \%)$. Median days to neutrophil and platelet recovery were 21 (range, 10-75) and 34 (range, 16-399), respectively. Conditioning regimens and transplant features are shown in Table 1. Median follow up was 44 months (range, 13-71, with 13 transplants performed in the last two years of the study period). OS and EFS at 36 months were $71 \%(55-87 \%)$ and $45 \%(45-63 \%)$, respectively (Figure $1 \mathrm{~A}$ and $\mathrm{B})$. Ten patients died; death was due to GvHD $(n=6)$, infection $(n=3)$, or multi-organ failure $(n=1)$. Patients who were transplanted from unrelated donor (UD) had an inferior outcome compared with those engrafted from matched sibling donors (MSD) [OS 51\% (range, 26-76\%) vs. 92\% (range, $76-100 \%$ ); $P=0.05]$. OS of patients with iron overload was significantly worse [45\% (range, 18-2\%) vs. $90 \%$ (range, $76-100 \%$ ) $P=0.007]$ compared with those transplanted without (Figure 1C and D). There was no difference in survival between patients transplanted before and after 2010, or between patients conditioned with or without fludarabine. The incidence of graft failure in patients transplanted with and without iron overload was $17 \%$ (range, $0-38 \%$ ) and $6 \%$ (range, $0-17 \%$ ), respectively $(P=0.382)$. Graft failure was $6 \%$ (range, 0 $17 \%$ ) and $16 \%$ (range, $0-32 \%$ ) in patients transplanted from MSD and UD, respectively $(P=0.497)$. Of note, this probability, due to competitive causes, was significantly higher in patients transplanted with versus without iron overload ( $59 \%$ vs. $17 \% ; P=0.024)$ and from a UD versus a MSD, respectively (55\% vs. $31 \% ; P=0.05$ ) (Figure $1 \mathrm{E}$ and F). A trend towards a higher incidence of grade II-IV aGvHD was also seen in patients transplanted from a UD 
(59\% vs. $25 \% ; P=0.06)$ and with iron overload (53\% vs. $25 \% ; P=0.1)$, as compared to those engrafted from MSD and with no iron overload, respectively. Severe (Grade III-IV) aGvHD incidence was $29 \%$, and was significantly higher $(P=0.005)$ in UD $(53 \%)$ than in MSD transplants $(10 \%)$.

To the best of our knowledge, this is the largest reported cohort of patients transplanted for CDA. Previous publications have been anecdotal experiences including a total of thirteen children, composed of CDA type $\mathrm{I},{ }^{9}$ type II, ${ }^{10-13}$ and type III, ${ }^{16}$ transplanted from sibling donors in all cases except for three patients who were transplanted from a UD. ${ }^{11,12,16}$ of particular note, our study, which included $44 \%$ of patients transplanted from a UD, showed that donor type still affects the outcome since MSD SCT patients have superior OS and engraftment.

Another factor affecting the outcome of our cohort was iron overload, which is an intrinsic feature of patients with CDA due to hepcidin downregulation and ineffective erythropoiesis. We also found an association between iron overload and GvHD that might become fully significant if a larger number of patients was available. Previous reports on thalassemia ${ }^{17,18}$ showed that tissue damage secondary to iron accumulation may enhance the risk of transplant-related toxicity and predispose patients to a higher incidence of aGvHD. This outlines the importance of a pre-transplant definition of the risk the patients may incur, which generated a dramatic improvement in the outcome in the case of thalassemia patients (Pesaro risk classes). ${ }^{17}$ The lack of such a pre-SCT selection in CDA patients might explain their worse outcome compared with other transfusion-dependent, hemolytic anemias like the thalassemias; indeed, the better outcome of CDA patients transplanted without iron overload seems to support this hypothesis. Amongst the risk factors, iron overload in CDA candidates for SCT needs to be carefully evaluated and reduced with effective chelation.

Graft rejection of this CDA cohort was comparable to that seen in thalassemia patients ${ }^{19}$ and was negatively affected by donor type (worse with UD) and iron overload. This points to the need for careful evaluation of the choice of donor type in the decision-making process.

Most patients were conditioned with myeloablative regimens including busulfan/treosulfan. In the limited number of cases included in this study, no difference was noted compared to patients who, in addition, received fludarabine or cyclophosphamide. Of note, the only patients of our cohort who received a non-myeloablative conditioning regimen had a successful outcome, as did the only previously reported CDA subject, ${ }^{16}$ who was conditioned with fludarabine-cyclophosphamide and a-day course of total body irradiation. This raises the issue of the appropriateness of reduced-intensity conditioning in CDA. Unfortunately, we could not address this aspect in this study due to the lack of available data, but it might be worth investigating in future analyses.

In conclusion, despite the lower overall outcome compared to previous reports on thalassemia patients, SCT may represent an alternative therapeutic option in CDA, with outcomes appearing to be superior in patients transplanted from MSD and without iron overload. Indeed, iron overload is an important factor that needs to be controlled and eventually treated before transplant. In this respect, CDA patients might benefit from pre-SCT risk assessment, e.g. like the Pesaro criteria, which may have a favorable impact on the overall outcome. The high incidence of graft failure and aGvHD, and the lower overall survival observed in patients transplanted from UD, should be taken into consideration and discussed with parents if a familial donor is not available.

Maurizio Miano, ${ }^{1}$ Dirk-Jan Eikema, ${ }^{2}$ Mahmoud Aljurf,

Pieter J. van't Veer, ${ }^{4}$ Gülyüz Öztürk, ${ }^{5}$ Matthias Wölfl,

Frans Smiers, Angsar Schulz, Gerard Socie, ${ }^{7}$

Kim Vettenranta, ${ }^{10}$ Cristina Diaz de Heredia,

Marco Zecca, ${ }^{12}$ Johan Maertens, ${ }^{13}$ Montserrat Rovira, ${ }^{14}$

Jorge Sierra, ${ }^{15}$ Duygu Uckan-Cetinkaya, ${ }^{16}$

Elena Skorobogatova, ${ }^{17}$ Ali Bülent Antmen, ${ }^{18}$ Jean-Hugues

Dalle, ${ }^{19}$ Miroslaw Markiewicz, ${ }^{20}$ Rose Marie Hamladji, ${ }^{21}$

Vassiliki Kitra-Roussou, ${ }^{22}$ Giorgio La Nasa, ${ }^{23}$

Gergely Kriván, ${ }^{24}$ Amal Al-Seiraihy, ${ }^{3}$ Stefano Giardino, ${ }^{25}$

Antonio Maria Risitano, ${ }^{26}$ Regis Peffault de Latour ${ }^{9}$

and Carlo Dufour'

${ }^{1}$ Hematology Unit, IRCCS Istituto Giannina Gaslini, Genoa, Italy; ${ }^{2}$ EBMT Statistical Unit, Leiden, Leiden, the Netherlands; ${ }^{3}$ King Faisal Specialist Hospital and Research Centre, Riyadh, Saudi Arabia; ${ }^{4}$ EBMT Data Office Leiden, Leiden, the Netherlands; 'Acibadem University Atakent Hospital, Istanbul, Turkey; 'University Children's Hospital, Würzburg, Würzburg, Germany; Leiden University Hospital, Leiden, the Netherlands; ${ }^{8}$ Klinik für Kinder-und Jugend medizin Ulm, Ulm, Germany; ${ }^{9}$ Hopital St. Louis, Paris, France; ${ }^{10}$ University of Helsinki, Helsinki, Finland; " Hospital Vall d'Hebron, Barcelona, Spain; ${ }^{12}$ Fondazione IRCCS Policlinico San Matteo, Pavia, Italy; ${ }^{13}$ University Hospital Gasthuisberg, Leuven, Belgium; ${ }^{14}$ Hospital Clinic Institute of Hematology and Oncology, Barcelona, Spain, ${ }^{15}$ Hospital Santa Creu I Sant Pau, Barcelona, Spain; ${ }^{16}$ Hacettepe University Children's Hospital, Ankara, Turkev; ${ }^{17}$ The Russian Children's Research Hospital, Moscow, Russia; ${ }^{18}$ Adana Acibadem Hospital, Adana, Turkey; ${ }^{19}$ Hôpital Robert Debre, Paris, France; ${ }^{20}$ Medical University of Silesia, Katowice, Poland; ${ }^{21}$ Centre Pierre et Marie Curie, Alger, Algeria; ${ }^{22}$ St. Sophia's Children's Hospital, Athens, Greece; ${ }^{23}$ Centro Trapianti di Midollo Osseo, Cagliari, Italy; ${ }^{24}$ Central Hospital of Southern Pest-National Institute of Hematology and Infectious Disease, Budapest, Hungary; ${ }^{25}$ Stem Cell Transplantation Unit, IRCCS Istituto Giannina Gaslini, Genoa, Italy and ${ }^{26}$ Federico II University, Naples, Italy

Correspondence: MAURIZIO MIANO

mauriziomiano@gaslini.org.

doi:10.3324/haematol.2018.206623

Information on authorship, contributions, and financial \& other disclosures was provided by the authors and is available with the online version of this article at wWw. haematologica.org.

\section{References}

1. Wickramasinghe SN, Wood WG. Advances in the understanding of the congenital dyserythropoietic anemias. Br J Haematol. 2005;131(4):431-446.

2. Gambale A, Iolascon A, Andolfo I, Russo R. Diagnosis and management of congenital dyserythropoietic anemias. Exp Rev Hematol 2016;9(3):283-296

3. Iolascon A, Delaunay J, Wickramasinghe SN, et al. Natural history of congenital dyserythropoietic anemia type II. Blood. 2001:98(4):12581260 .

4. Tamary $\mathrm{H}$, Offret $\mathrm{H}$, Dgany $\mathrm{O}$, et al. Congenital dyserythropoietic anemia, type $\mathrm{I}$, in a Caucasian patient with retinal angioid streaks (homozygous Arg1042Trp mutation in codanin-1). Eur J Haematol. 2008;80(3):271-274.

5. Russo R, Gambale A, Esposito MR, et al. Two founder mutations in the SEC23B gene account for the relatively high frequency of CDA II in the Italian population. Am J Hematol. 2011;86(9):727-732.

6. Lavabre-Bertrand T, Ramos J, Delfour C, et al. Long-term alpha interferon treatment is effective on anaemia and significantly reduces iron overload in congenital dyserythropoiesis type I. Eur J Haematol. 2004;73(5):380-383.

7. Goede JS, Benz R, Fehr J, Schwarz K, Heimpel H. Congenital dyserythropoietic anemia type I with bone abnormalities, mutations of the CDAN I gene, and significant responsiveness to alpha-interferon therapy. Ann Hematol. 2006:85(9):591-595

8. Choudhry VP, Saraya AK, Kasturi J, Rath PK. Congenital dyserythro- 
poietic anemias: splenectomy as a mode of therapy. Acta Haematol. 1981;66(3):195-201.

9. Ayas M, Al-Jefri A, Baothman A, et al. Transfusion-dependent congenital dyserythropoietic anemia type I successfully treated with allogeneic stem cell transplantation. Bone Marrow Transpl. 2002:29(8):681-682.

10. Unal S, Russo R, Gumruk F, et al. Successful hematopoietic stem cell transplantation in a patient with congenital dyserythropoietic anemia type II. Pediatr Transplant. 2014;18(4):E130-133.

11. Braun $M$, Wölfl $M$, Wiegering V, et al. Successful treatment of an infant with CDA type II by intrauterine transfusions and postnatal stem cell transplantation. Pediatr Blood Cancer. 2014;61(4):743-745.

12. Buchbinder D, Nugent D, Vu D, et al. Unrelated hematopoietic stem cell transplantation in a patient with congenital dyserythropoietic anemia and iron overload. Pediatr Transplant. 2012;16(3):E69-73.

13. Modi G, Shah S, Madabhavi I, et al. Succesful allogeneic hematopoietic stem cell transplantation of a patient suffering from type II congenital dyserythropoietic anemia a rare case report from Western India. Case Rep Hematol. 2015;2015:792485.

14. Remacha AF, Badell I, Pujol-Moix N, et al. Hydrops fetalis-associated congenital dyserythropoietic anemia treated with intrauterine trans- fusions and bone marrow transplantation. Blood. 2002;100(1):356358.

15. Iolascon A, Sabato V, de Mattia D, Locatelli F. Bone marrow transplantation in a case of severe, Type II congenital dyserythropoietic anemia (CDA II). Bone Marrow Transpl. 2001;27(2):213-215.

16. Oh A, Patel PR, Aardsma N, et al. Non-myeloablative allogeneic stem cell transplant with post-transplant cyclophosphamide cures the first adult patient with congenital dyserythropoietic anemia. Bone Marrow Transpl. 2017;52(6):905-906.

17. Luccarelli G, Galimberti M, Polchi P, et al. Bone Marrow Transplantation in patients with thalassemia. N Eng J Med. 1990; 322(7):417-421.

18. Luccarelli G, Clift RA, Galimberti M, et al. Bone Marrow Transplantation in adult thalassemic patients. Blood. 1999; 93(4);1164-1167.

19. Angelucci E, Matthes-Martin S, Baronciani D, et al; EBMT Inborn Error and EBMT Pediatric Working Parties. Hematopoietic stem cell transplantation in thalassemia major and sickle cell disease: indications and management recommendations from an international expert panel Haematologica. 2014;99(5):811-820. 